\title{
UPPER BOUNDS FOR THE MAXIMUM OF A RANDOM WALK WITH NEGATIVE DRIFT
}

\author{
JOHANNES KUGLER *** AND \\ VITALI WACHTEL, ${ }^{* * * *}$ University of Munich
}

\begin{abstract}
Consider a random walk $S_{n}=\sum_{i=0}^{n} X_{i}$ with negative drift. This paper deals with upper bounds for the maximum $M=\max _{n \geq 1} S_{n}$ of this random walk in different settings of power moment existences. As is usual for deriving upper bounds, we truncate summands. Therefore, we use an approach of splitting the time axis by stopping times into intervals of random but finite length and then choose a level of truncation on each interval. Hereby, we can reduce the problem of finding upper bounds for $M$ to the problem of finding upper bounds for $M_{\tau}=\max _{n \leq \tau} S_{n}$. In addition we test our inequalities in the heavy traffic regime in the case of regularly varying tails.
\end{abstract}

Keywords: Limit theorem; random walk; renewal theorem

2010 Mathematics Subject Classification: Primary 60G50

Secondary 60G52

\section{Introduction and statement of results}

Let $\left\{S_{n}, n \geq 0\right\}$ denote the random walk with increments $X_{i}$, that is,

$$
S_{0}:=0, \quad S_{n}:=\sum_{i=1}^{n} X_{i}, \quad n \geq 1 .
$$

We shall assume that $X_{1}, X_{2}, \ldots$ are independent copies of a random variable $X$ with distribution function $F$ and $a:=-\mathbb{E}[X]>0$. The random walk $S_{n}$ drifts to $-\infty$ and the total maximum $M:=\max _{k \geq 0} S_{k}$ is finite almost surely. The random variable $M$ plays a crucial role in a number of applications. For example, its distribution coincides with the stationary distribution of the queue length in simple queueing systems. Another important application comes from the mathematics of insurance: under some special restrictions on $X$ the quantity $\mathbb{P}(M>u)$ is equal to the ruin probability in the so-called renewal arrivals model.

The tail behaviour of $M$ has been studied extensively in the literature. The first result goes back, apparently, to Cramér and Lundberg (see, e.g. Asmussen [2]): If

$$
\mathbb{E}\left[\mathrm{e}^{h_{0} X}\right]=1 \text { for some } h_{0}>0,
$$

and, in addition, $\mathbb{E}\left[X \mathrm{e}^{h_{0} X}\right]<\infty$, then there exists a constant $c_{0} \in(0,1)$ such that

$$
\mathbb{P}(M>x) \sim c_{0} \mathrm{e}^{-h_{0} x} \quad \text { as } x \rightarrow \infty .
$$

Received 13 December 2011; revision received 7 December 2012.

Supported by the DFG.

* Postal address: Mathematical Institute, University of Munich, Theresienstrasse 39, D-80333, Munich, Germany.

** Email address: johannes.kugler@mathematik.uni-muenchen.de

*** Email address: vitali.wachtel@ mathematik.uni-muenchen.de 
The case $\mathbb{E}\left[X \mathrm{e}^{h_{0} X}\right]=\infty$ has been considered recently by Korshunov [12] and the way in which a high value in this setting is attained was studied later by Foss and Puhalskii [7].

If (1) is not fulfilled, then one assumes that the distribution of $X$ is regular in some sense. To specify what regular means we recall some definitions and known properties. For their proofs we refer the reader to [9]. Consider a distribution function $B$ on $\mathbb{R}$ and let $\bar{B}(x)=1-B(x)$ be the right tail of $B$. A distribution function $B$ with support $\mathbb{R}_{+}$is called subexponential, if $\bar{B}(x)>0$ for all $x$ and

$$
\lim _{x \rightarrow \infty} \frac{\overline{B^{*} n}(x)}{\bar{B}(x)}=n
$$

for all $n \geq 2$, where $B^{* n}(x)$ is the $n$-fold convolution of $B$ with itself. For the subexponentiality it is sufficient to verify the equation (2) in the case $n=2$. All subexponential distributions are heavy tailed, i.e. $\mathbb{E}[\exp (\varepsilon X)]=\infty$ for all $\varepsilon>0$, hence subexponential distributions do not satisfy (1). If (1) is not fulfilled, the most classical result for the asymptotics of $M$ is due to Veraverbeke [18], who showed that if the integrated tail $\bar{G}(x):=\int_{x}^{\infty} \bar{F}(u) \mathrm{d} u$ is subexponential, then

$$
\mathbb{P}(M>x) \sim \frac{1}{a} \bar{G}(x) \quad \text { as } x \rightarrow \infty .
$$

In many situations one needs nonasymptotic properties of the distribution of $M$. Since the exact form of that distribution is known in some special cases only, good estimates are required. Under condition (1) we have, for all $x>0$, the so-called Lundberg inequality

$$
\mathbb{P}(M>x) \leq \mathrm{e}^{-h_{0} x}
$$

In the case when (1) is not fulfilled, upper bounds for $\mathbb{P}(M>x)$ have been derived by Kalashnikov [11] and by Richards [17]. The approach in these papers is based on the representation of $M$ as a geometric sum of independent random variables:

$$
\mathbb{P}(M>x)=\sum_{k=0}^{\infty} q(1-q)^{k} \mathbb{P}\left(\chi_{1}^{+}+\chi_{2}^{+} \cdots+\chi_{k}^{+}>x\right),
$$

where $\left\{\chi_{k}^{+}\right\}$are independent random variables and $q=\mathbb{P}(M=0)$. The main difficulty with this approach is the fact that one has to know the distribution of $\chi_{k}^{+}$and the parameter $q$. In some special cases this information can be obtained from the initial data. But in general we have to obtain appropriate estimates for $q$ and $\mathbb{P}\left(\chi_{1}^{+}>x\right)$.

The main purpose of the present paper is to derive upper bounds for $\mathbb{P}(M>x)$ assuming the existence of power moments of $X$ only. Thereby we want to avoid the representation via geometric sums and use a supermartingale construction instead.

As is usual for deriving upper bounds, we are going to truncate summands and to use inequalities, which are based on truncated exponential moments. But the problem is that we have infinitely many $X_{i}$ s, so we cannot truncate all of them at the same level. Thus, we have to split the time axis into intervals of finite length and then choose a level of truncation on each of these intervals. Take, for example, a deterministic strictly increasing sequence $k_{n}$ with $k_{0}=0$ 
and consider the intervals $I_{n}:=\left(k_{n}, k_{n+1}\right]$, giving

$$
\begin{aligned}
\mathbb{P}(M \geq x) & =\mathbb{P}\left(\bigcup_{k \geq 0}\left\{S_{k} \geq x\right\}\right) \\
& \leq \sum_{n=0}^{\infty} \mathbb{P}\left(\bigcup_{k \in I_{n}}\left\{S_{k} \geq x\right\}\right) \\
& \leq \sum_{n=0}^{\infty} \mathbb{P}\left(\max _{k \leq k_{n+1}}\left(S_{k}-k a\right) \geq x-k_{n} a\right) .
\end{aligned}
$$

Now, one can apply the Fuk-Nagaev inequalities, see [15], to every probability in the last line. It is clear that replacing $\sup _{k \in I_{n}}\left(S_{k}-k a\right)$ by $\sup _{k \leq k_{n+1}}\left(S_{k}-k a\right)$ is not too rough if and only if $k_{n+1}$ and $k_{n+1}-k_{n}$ are comparable. Thus, we have to take $k_{n}$ such that it grows exponentially. Using this approach with $k_{n}=x 2^{n}$, Borovkov [3] obtained a version of the Markov inequality for $M$.

Our strategy, however, is quite different and consists of splitting $[0, \infty)$ into random intervals defined by a sequence of stopping times. More precisely, we introduce the stopping time

$$
\tau_{z}:=\min \left\{k \geq 0: S_{k} \leq-z\right\}, \quad z \geq 0 .
$$

Let $M_{\tau}=\max _{1 \leq k \leq \tau_{z}} S_{k}$. We split the tail probability

$$
\mathbb{P}(M>x) \leq \mathbb{P}\left(M_{\tau_{z}}>x\right)+\mathbb{P}\left(\max _{k \geq \tau_{z}} S_{k}>x\right)
$$

and consider the continuation of the process $\left(S_{k}\right)$ beyond $\tau_{z}$ as a probabilistic replica of the entire process. By $S_{\tau_{z}} \leq-z$ follows

$$
\mathbb{P}\left(\max _{k \geq \tau_{z}} S_{k}>x\right) \leq \mathbb{P}(M>x+z) .
$$

As a result, we have

$$
\mathbb{P}(M>x) \leq \mathbb{P}\left(M_{\tau}>x\right)+\mathbb{P}(M>x+z),
$$

and inductively we conclude

$$
\mathbb{P}(M>x) \leq \sum_{j=0}^{\infty} \mathbb{P}\left(M_{\tau}>x+j z\right) .
$$

It is worth mentioning that the difference between (5) and (7) is the same as between Riemann and Lebesgue integrals: We do not fit the random walk $S_{n}$ into a fixed splitting of the time, but choose the splitting depending on the paths of the random walk.

A decomposition similar to (6) has been used by Denisov [5] for deriving the asymptotics of $\mathbb{P}\left(M_{\tau_{0}}>x\right)$ from those of $\mathbb{P}\left(M \in\left[x, x-S_{\tau_{0}}\right)\right)$. In the present paper we use the opposite approach: we obtain estimates for $\mathbb{P}(M>x)$ from the ones for $\mathbb{P}\left(M_{\tau_{z}}>x\right)$.

We now state our results on $M_{\tau_{z}}$. 
Theorem 1. Assume that $A_{t}:=\mathbb{E}\left[|X|^{t}\right]<\infty$ for some $t \in(1,2]$. For all $y$ satisfying $y^{t-1} \geq(\mathrm{e}-1) A_{t} a^{-1}$ we have the following inequality:

$$
\begin{aligned}
\mathbb{P}\left(M_{\tau_{z}}>x\right) \leq & \frac{A_{t}^{x / y}}{a^{x / y-1}} \mathbb{E}\left[\tau_{z}\right] y^{-1-(t-1) x / y} \log \left(1+\frac{a y^{t-1}}{A_{t}}\right) \\
& +\left(1+\frac{A_{t}^{x / y}}{a^{x / y}} y^{-(t-1) x / y}\right) \mathbb{E}\left[\tau_{z}\right] \mathbb{P}(X>y)
\end{aligned}
$$

Remark 1. In the proof we show that (8) remains true if we replace $a$ and $A_{t}$ by $-\mathbb{E}[X,|X| \leq y]$ and $A_{t}(y)=\mathbb{E}\left[|X|^{t},|X| \leq y\right]$ respectively. In this case the restriction $y^{t-1}>(\mathrm{e}-1) a^{-1} A_{t}$ should be replaced by $\mathbb{E}[X,|X| \leq y]<0$. The use of truncated moments is more convenient in theoretical applications, but for deriving concrete estimates for $M$ it is easier to use full moments.

Let us now turn to the case $t>2$. Fix $\alpha \in(0,1)$ and put $\beta=1-\alpha$. We use the notation

$$
\psi_{1}(x):=\exp \left(\frac{2 \alpha a x}{\mathrm{e}^{t} \mathbb{E}\left[X^{2}\right]}\right)-1, \quad \psi_{2}:=\frac{\beta a}{A_{t,+}} .
$$

Theorem 2. Assume $\operatorname{var}(X)<\infty$ and $A_{t,+}:=\mathbb{E}\left[X^{t}, X>0\right]<\infty$ for some $t>2$.

(i) If y satisfies the condition

$$
\frac{2 \alpha a}{\mathrm{e}^{t} \mathbb{E}\left[X^{2}\right]} \leq \frac{1}{y} \log \left(1+\frac{\beta a}{A_{t,+}} y^{t-1}\right)
$$

then

$$
\mathbb{P}\left(M_{\tau_{z}}>x\right) \leq\left(1+\frac{1}{\psi_{1}(x)}\right) \mathbb{E}\left[\tau_{z}\right] \mathbb{P}(X>y)+\frac{2 \alpha a^{2} \mathbb{E}\left[\tau_{z}\right]}{\mathrm{e}^{t} \mathbb{E}\left[X^{2}\right] \psi_{1}(x)}
$$

(ii) If y satisfies the condition

$$
\frac{2 \alpha a}{\mathrm{e}^{t} \mathbb{E}\left[X^{2}\right]} \geq \frac{1}{y} \log \left(1+\frac{\beta a}{A_{t,+}} y^{t-1}\right)
$$

then

$$
\begin{aligned}
\mathbb{P}\left(M_{\tau_{z}}>x\right) \leq & \psi_{2}^{-x / y} \frac{\mathbb{E}\left[\tau_{z}\right]}{a} y^{-1-(t-1) x / y} \log \left(1+\psi_{2} y^{t-1}\right) \\
& +\left(1+\psi_{2}^{-x / y} y^{-(t-1) x / y}\right) \mathbb{E}\left[\tau_{z}\right] \mathbb{P}(X>y)
\end{aligned}
$$

Remark 2. With analogy to Theorem 1 , we can replace $\operatorname{var}(X)$ and $A_{t,+}$ by the corresponding truncated expectations $B^{2}(-\infty, x)=\mathbb{E}\left[X^{2}, X \leq y\right]$ and $A_{t,+}(y)=\mathbb{E}\left[X^{t}, X \in(0, y]\right]$ respectively.

Corollary 1. Assume that $\mathbb{P}(|X|>x)=L(x) x^{-r}$ for some $r>1$ and

$$
\frac{\mathbb{P}(X>x)}{\mathbb{P}(|X|>x)} \rightarrow p \in(0,1) \quad \text { as } x \rightarrow \infty .
$$

Then, it follows from (8) and (11) that

$$
\limsup _{x \rightarrow \infty} \frac{\mathbb{P}\left(M_{\tau_{z}}>x\right)}{\mathbb{P}(X>x)} \leq \mathbb{E}\left[\tau_{z}\right]
$$

for every $z>0$. 
But it follows from the results of Asmussen [1] (also see [5] and [8]) that

$$
\lim _{x \rightarrow \infty} \frac{\mathbb{P}\left(M_{\tau_{z}}>x\right)}{\mathbb{P}(X>x)}=\mathbb{E}\left[\tau_{z}\right]
$$

under the condition that the tail of $F$ is regularly varying. This means the inequalities (8) and (11) are asymptotically precise in the case of regularly varying tails.

In all these inequalities we have $\mathbb{E}\left[\tau_{z}\right]$ on the right hand side. It is hard to get an exact expression for this value via initial data, but there are good upper bounds in the literature: since $\mathbb{E}\left[\tau_{z}\right]<\infty$ (see, e.g. [6]) by Wald's identity,

$$
\mathbb{E}\left[\tau_{z}\right]=\frac{z+\mathbb{E}\left[R_{z}\right]}{a}
$$

where $R_{z}=-z-S_{\tau_{z}}$ denotes the overshoot in $\tau_{z}$. Hence, we get upper bounds for $\mathbb{E}\left[\tau_{z}\right]$ by the inequality of Lorden [13]: for $\mathbb{E}[X]<0$ and $\mathbb{E}\left[\left(X^{-}\right)^{2}\right]<\infty$,

$$
\mathbb{E}\left[R_{z}\right] \leq \frac{\mathbb{E}\left[\left(X^{-}\right)^{2}\right]}{a},
$$

and by the inequality of Mogul'skii [14]: for $\mathbb{E}[X] \leq 0$ and $\mathbb{E}\left[|X|^{3}\right]<\infty$,

$$
\mathbb{E}\left[R_{z}\right] \leq A \frac{3}{2} \frac{\mathbb{E}\left[|X|^{3}\right]}{\mathbb{E}\left[X^{2}\right]},
$$

where $A$ is a certain constant, $A \leq 2$. The disadvantage of these bounds is that we have to assume the existence of the second or even the third moment. We give another bound, which only requires the finiteness of the moment of order $t, t \in(1,2]$.

Proposition 1. Assume that $A_{t,-}:=\mathbb{E}\left[\left(X^{-}\right)^{t}\right]<\infty$ for some $t \in(1,2]$, then, for every $z>0$,

$$
\mathbb{E}\left[R_{z}\right] \leq \frac{t^{t /(t-1)} A_{t,-}^{1 /(t-1)}}{(t-1) a^{t /(t-1)}}\left(\mathbb{E}[-X, X<0]+\frac{z^{2-t}}{t} A_{t,-}\right) .
$$

Combining (12) with (13), (14), or (15) we obtain upper bounds for $\mathbb{E}\left[\tau_{z}\right]$. Substituting these bounds into the inequalities in Theorems 1 and 2 we get bounds for $\mathbb{P}\left(M_{\tau}>x\right)$, which contain information on $X$ only. So, they can be used for concrete calculations.

We now come back to the global maximum.

Theorem 3. Fix some $\theta \in(0,1)$ and define

$$
\begin{array}{cc}
c_{1}:=\frac{3 A_{t}^{1 / \theta} \theta^{-(t-1) / \theta}}{(t-1) a^{1 / \theta-1}}, & c_{2}:=\frac{3 A_{t,+}^{1 / \theta} \theta^{-(t-1) / \theta}}{(t-1) \beta^{1 / \theta} a^{1 / \theta-1}}, \\
\psi_{3}(x):=\frac{a \theta^{t-1} x^{t-1}}{A_{t}}, & \psi_{4}(x):=\frac{\beta a \theta^{t-1} x^{t-1}}{A_{t,+}} .
\end{array}
$$

(i) Assume that $A_{t}<\infty$ for some $t \in(1,2]$. Then, for every $x$ satisfying $x^{t-1} \geq \theta^{1-t}(\mathrm{e}-$ 1) $A_{t} a^{-1}$ and $x \geq z(t-1) \theta^{-1}$, we have

$$
\begin{aligned}
\mathbb{P}(M>x) \leq & c_{1} \frac{\mathbb{E}\left[\tau_{z}\right]}{z} \log \left(1+\psi_{3}(x)\right) x^{-(t-1) / \theta} \\
& +\left(1+\psi_{3}(x)^{-1 / \theta}\right) \mathbb{E}\left[\tau_{z}\right]\left(\frac{1}{\theta z} \bar{G}(\theta x)+\mathbb{P}(X>\theta x)\right) .
\end{aligned}
$$


(ii) Assume that $\operatorname{var}(X)<\infty$ and $A_{t,+}<\infty$ for some $t>2$. Then, for every $x$ satisfying (10) for $y=\theta x$ and the conditions $x^{t-1} \geq \theta^{1-t}\left(\mathrm{e}^{\theta}-1\right) A_{t,+} \beta^{-1} a^{-1}$ and $x \geq z(t-1) \theta^{-1}$, we have

$$
\begin{aligned}
\mathbb{P}(M>x) \leq & c_{2} \frac{\mathbb{E}\left[\tau_{z}\right]}{z} \log \left(1+\psi_{4}(x)\right) x^{-(t-1) / \theta} \\
& +\left(1+\psi_{4}(x)^{-1 / \theta}\right) \mathbb{E}\left[\tau_{z}\right]\left(\frac{1}{\theta z} \bar{G}(\theta x)+\mathbb{P}(X>\theta x)\right) .
\end{aligned}
$$

Corollary 2. If the assumptions of Corollary 1 hold, then it follows from Theorem 3 that

$$
\limsup _{x \rightarrow \infty} \frac{\mathbb{P}(M>x)}{\bar{G}(x)} \leq \frac{\mathbb{E}\left[\tau_{z}\right]}{z} \theta^{-r} .
$$

Since the left-hand side does not depend on $\theta$ and $z$, we can let $\theta \rightarrow 1$ and $z \rightarrow \infty$. Noting that each of (13) and (14) combined with (12) yields

$$
\frac{\mathbb{E}\left[\tau_{z}\right]}{z} \rightarrow \frac{1}{a} \quad \text { as } z \rightarrow \infty
$$

we conclude that

$$
\limsup _{x \rightarrow \infty} \frac{\mathbb{P}(M>x)}{\bar{G}(x)} \leq \frac{1}{a} .
$$

Comparing this with (3) we see that the inequalities in Theorem 3 are asymptotically precise. This even remains valid if we bound $\mathbb{E}\left[\tau_{z}\right]$ in the inequalities of Theorem 3 by combining (13) or (14) with (12).

The reason why we are able to obtain asymptotically precise bounds is because we may choose $z$ to be arbitrarily large. That possibility seems to be a quite important advantage of our method compared to geometric sums. If the distribution of $\chi_{1}^{+}$is subexponential, then it follows easily from (4) that

$$
\mathbb{P}(M>x) \sim\left(\frac{1}{q}-1\right) \mathbb{P}\left(\chi_{1}^{+}>x\right) \quad \text { as } x \rightarrow \infty .
$$

Therefore, in order to obtain an upper bound for the maximum we need to control the quantity $1 / q$. It is well known that $1 / q=\mathbb{E}\left[-S_{\tau_{0}}\right]=\mathbb{E}\left[R_{0}\right]$. Thus, we may apply (13), (14), or (15) with $z=0$. But corresponding inequalities for $M$ will not be asymptotically precise. Summarising, the approach via geometric sums can only lead to asymptotically precise bounds if $q$ is known.

We next test our inequalities in the heavy-traffic regime. Let $\left\{S^{(a)}, a \geq 0\right\}$ be a family of random walks with $\mathbb{E}\left[X^{(a)}\right]=-a$. We assume that $X^{(a)}=X^{(0)}-a$ for all $a>0$. Let $M^{(a)}$ denote the corresponding maximum. It is known that if $X^{(0)}$ belongs to the domain of attraction of a stable law, then there exists a regularly varying function $g(a)$ such that $g(a) M^{(a)}$ converges weakly as $a \rightarrow 0$. It turns out that our inequalities may be applied to large deviation problems in the heavy-traffic convergence mentioned above. More precisely, they give asymptotically precise bounds for the probabilities $\mathbb{P}\left(M^{(a)}>x_{a}\right)$ if $x_{a} \gg 1 / g(a)$. In the case of $\sigma^{2}:=\operatorname{var}\left(X^{(0)}\right)$ being finite, we have $g(a)=a$ and the weak limit of $a M^{(a)}$ is the exponential distribution with parameter $2 / \sigma^{2}$.

Theorem 4. Assume that $\sigma^{2}<\infty$ and the right tail of the distribution function of $X^{(0)}$ is regularly varying with index $r>2$, that is, $\mathbb{P}\left(X^{(0)}>u\right)=u^{-r} L(u)$, where $L$ is slowly varying. If

$$
\liminf _{a \rightarrow 0} \frac{x_{a}}{a^{-1} \log a^{-1}}>\mathrm{e}^{r} \frac{(r-2)}{2} \sigma^{2}
$$


then

$$
\mathbb{P}\left(M^{(a)}>x_{a}\right) \sim \frac{x_{a}^{-r+1} L\left(x_{a}\right)}{(r-1) a} \text { as } a \rightarrow 0 .
$$

Olvera-Cravioto et al. [16] have shown that, for an $M / G / 1$ queue, the relation (19) holds under the condition

$$
\liminf _{a \rightarrow 0} \frac{x_{a}}{a^{-1} \log a^{-1}}>\frac{(r-2)}{2} \sigma^{2} .
$$

We believe that the latter condition should also be sufficient for the validity of (19) in the general case. The extra factor $\mathrm{e}^{r}$ appears in (18) only as a consequence of the technique we used, and can be removed by adapting (17) to the heavy-traffic setting.

Theorem 5. Assume that $\mathbb{E}\left[\left(\min \left\{0, X^{(0)}\right\}\right)^{2}\right]<\infty$ and $\mathbb{P}\left(X^{(0)}>u\right)=u^{-r} L(u)$ with $r \in$ $(1,2)$. If

$$
\liminf _{a \rightarrow 0} g(a) x_{a}=\infty
$$

then

$$
\mathbb{P}\left(M^{(a)}>x_{a}\right) \sim \frac{x_{a}^{-r+1} L\left(x_{a}\right)}{(r-1) a} \text { as } a \rightarrow 0 .
$$

We have imposed the condition $\mathbb{E}\left[\left(\min \left\{0, X^{(0)}\right\}\right)^{2}\right]<\infty$ just to use the Lorden inequality for the overshoot. If we replace that condition by $\mathbb{E}\left[\left(\min \left\{0, X^{(0)}\right\}\right)^{t}\right]<\infty$ with $t \in(1,2)$, then, using Proposition 1, we can show that (21) holds for $x_{a} \gg a^{-t /(t-1)^{2}}$ only. The reason is the roughness of Proposition 1 for small values of $a$. Indeed, if we use (15) even with $t=2$, we get the bound $\mathbb{E}\left[R_{z}\right] \leq \mathrm{Ca}^{-2}$, which is much worse than the Lorden inequality.

\section{Proofs}

\subsection{Proofs of Theorems 1 and 2}

We set for brevity $\tau=\tau_{z}$.

Lemma 1. For all h satisfying

$$
\mathbb{E}\left[\mathrm{e}^{h X}, X \leq y\right] \leq 1
$$

we have the inequality

$$
\mathbb{P}\left(M_{\tau}>x\right) \leq\left(1+\frac{1}{\mathrm{e}^{h x}-1}\right) \mathbb{E}[\tau] \mathbb{P}(X>y)+\mathbb{E}[\tau] \frac{a h}{\mathrm{e}^{h x}-1}
$$

Proof. Our strategy is to truncate the random variables $X_{i}$ in the level $y$ :

$$
\begin{aligned}
\mathbb{P}\left(M_{\tau}>x\right) & \leq \mathbb{P}\left(M_{\tau}>x, \max _{1 \leq k \leq \tau} X_{k} \leq y\right)+\mathbb{P}\left(\max _{1 \leq k \leq \tau} X_{k}>y\right) \\
& =\mathbb{P}\left(M_{\tau} \mathbf{1}_{\left\{\max _{1 \leq k \leq \tau} X_{k} \leq y\right\}}>x\right)+\mathbb{P}\left(\max _{1 \leq k \leq \tau} X_{k}>y\right) .
\end{aligned}
$$

From the Wald identity follows

$$
\mathbb{P}\left(\max _{1 \leq k \leq \tau} X_{k}>y\right) \leq \mathbb{E}\left[\sum_{k=1}^{\tau} \mathbf{1}_{\left\{X_{k}>y\right\}}\right]=\mathbb{E}[\tau] \mathbb{P}(X>y) .
$$


To examine the first term on the right-hand side of (24) we introduce the process $\left\{W_{k}\right\}$ defined by

$$
W_{0}:=1, \quad W_{k}:=\prod_{i=1}^{k} \mathrm{e}^{h X_{i}} \mathbf{1}_{\left\{X_{i} \leq y\right\}}, \quad k \geq 1 .
$$

It is clear that if $h$ satisfies (22), $\left\{W_{k}\right\}$ is a positive supermartingale. Define

$$
\sigma_{y}:=\min \left\{k \geq 1: X_{k}>y\right\}, \quad t_{x}:=\min \left\{k \geq 1: S_{k}>x\right\}, \quad T:=\min \left\{\sigma_{y}, t_{x}, \tau\right\} .
$$

Applying the optional stopping theorem to the supermartingale $\left\{W_{k \wedge T}\right\}$, we have

$$
1=W_{0} \geq \mathbb{E}\left[W_{T}\right]=\mathbb{E}\left[W_{T} \mathbf{1}_{\left\{t_{x}<\tau, t_{x}<\sigma_{y}\right\}}\right]+\mathbb{E}\left[W_{T} \mathbf{1}_{\left\{\tau<t_{x}, \tau<\sigma_{y}\right\}}\right] .
$$

We analyse the two terms on the right-hand side separately:

$$
\mathbb{E}\left[W_{T} \mathbf{1}_{\left\{t_{x}<\tau, t_{x}<\sigma_{y}\right\}}\right] \geq \mathrm{e}^{h x} \mathbb{P}\left(t_{x}<\tau<\sigma_{y}\right)=\mathrm{e}^{h x} \mathbb{P}\left(M_{\tau} \mathbf{1}_{\left\{\max _{1 \leq k \leq \tau} X_{k} \leq y\right\}}>x\right)
$$

and

$$
\begin{aligned}
\mathbb{E}\left[W_{T}\right. & \left.\mathbf{1}_{\left\{\tau<t_{x}, \tau<\sigma_{y}\right\}}\right] \\
& =\mathbb{E}\left[\mathrm{e}^{h S_{\tau}}\right]-\mathbb{E}\left[\mathrm{e}^{h S_{\tau}} \mathbf{1}_{\left\{M_{\tau}>x\right\} \cup\left\{\max _{1 \leq k \leq \tau} X_{k}>y\right\}}\right] \\
& \geq \mathbb{E}\left[\mathrm{e}^{h S_{\tau}}\right]-\mathrm{e}^{-h z}\left(\mathbb{P}\left(M_{\tau} \mathbf{1}_{\left\{\max _{1 \leq k \leq \tau} X_{k} \leq y\right\}}>x\right)+\mathbb{P}\left(\max _{1 \leq k \leq \tau} X_{k}>y\right)\right) .
\end{aligned}
$$

Consequently,

$$
\mathbb{P}\left(M_{\tau} \mathbf{1}_{\left\{\max _{1 \leq k \leq \tau} X_{k} \leq y\right\}}>x\right) \leq \frac{1-\mathbb{E}\left[\mathrm{e}^{h S_{\tau}}\right]+\mathbb{P}\left(\max _{1 \leq k \leq \tau} X_{k}>y\right)}{\mathrm{e}^{h x}-1}
$$

and, hence, by applying (25),

$$
\mathbb{P}\left(M_{\tau} \mathbf{1}_{\left\{\max _{1 \leq k \leq \tau} X_{k} \leq y\right\}}>x\right) \leq \frac{1-\mathbb{E}\left[\mathrm{e}^{h S_{\tau}}\right]+\mathbb{E}[\tau] \mathbb{P}(X>y)}{\mathrm{e}^{h x}-1} .
$$

It is easy to see that

$$
\mathbb{E}\left[\mathrm{e}^{h S_{\tau}}\right] \geq \mathbb{E}\left[1+h S_{\tau}\right]=1+h \mathbb{E}\left[S_{\tau}\right]
$$

and as a result we have

$$
\mathbb{P}\left(M_{\tau} \mathbf{1}_{\left\{\max _{1 \leq k \leq \tau} X_{k} \leq y\right\}}>x\right) \leq \mathbb{E}[\tau] \frac{a h+\mathbb{P}(X>y)}{\mathrm{e}^{h x}-1} .
$$

Applying (25) and (26) to the summands in (24) completes the proof.

To prove Theorems 1 and 2 we need to choose a specific $h$ for which (22) holds. The optimal choice would be the positive solution of the equation $\mathbb{E}\left[\mathrm{e}^{h X}, X \leq y\right]=1$, which is in the spirit of the Cramér-Lundberg condition. But it is not clear how to solve this equation. For this reason we replace $\mathbb{E}\left[\mathrm{e}^{h X}, X \leq y\right]=1$ by the equation $\phi(h, y)=1$, where $\phi(h, y)$ is an appropriate upper bound for $\mathbb{E}\left[\mathrm{e}^{h \bar{X}}, X \leq y\right]$.

If $A_{t}<\infty$, we may use a bound from the proof of Theorem 2 from [10], which says

$$
\mathbb{E}\left[\mathrm{e}^{h X}, X \leq y\right] \leq 1+h \mathbb{E}[X,|X| \leq y]+\frac{\mathrm{e}^{h y}-1-h y}{y^{t}} A_{t} .
$$


Using the Markov inequality we also obtain

$$
\mathbb{E}[X,|X| \leq y] \leq-a-\mathbb{E}[X, X \leq-y] \leq-a+\frac{A_{t}}{y^{t-1}},
$$

and, therefore,

$$
\mathbb{E}\left[\mathrm{e}^{h X}, X \leq y\right] \leq 1-h a+\frac{\mathrm{e}^{h y}-1}{y^{t}} A_{t} .
$$

Put $h_{0}:=(1 / y) \log \left(1+a y^{t-1} / A_{t}\right)$. It is easy to see that

$$
-h_{0} a+\frac{\mathrm{e}^{h_{0} y}-1}{y^{t}} A_{t} \leq 0
$$

for all $y$ such that $y^{t-1} \geq(\mathrm{e}-1) A_{t} a^{-1}$ and this implies that $h_{0}$ satisfies (22). Using (23) with $h=h_{0}$ and applying the inequality

$$
(1+u)^{x / y} \geq 1+u^{x / y}, \quad x \geq y,
$$

we obtain

$$
\begin{aligned}
\mathbb{P}\left(M_{\tau}>x\right) \leq & \frac{A_{t}^{x / y}}{a^{x / y-1}} \mathbb{E}[\tau] y^{-1-(t-1) x / y} \log \left(1+\frac{a y^{t-1}}{A_{t}}\right) \\
& +\left(1+\frac{A_{t}^{x / y}}{a^{x / y}} y^{-(t-1) x / y}\right) \mathbb{E}[\tau] \mathbb{P}(X>y) .
\end{aligned}
$$

Thus, the proof of Theorem 1 is complete.

In order to show that we can replace $\mathbb{E}[X]$ and $A_{t}$ by the corresponding truncated moments, see Remark 1, we first note that with analogy to (27) and by using $\mathrm{e}^{x}-1 \leq x \mathrm{e}^{x}$,

$$
\mathbb{E}\left[\mathrm{e}^{h X}, X \leq y\right] \leq 1+h \mathbb{E}[X,|X| \leq y]+h \frac{\mathrm{e}^{h y}-1}{y^{t-1}} \mathbb{E}\left[|X|^{t},|X| \leq y\right] .
$$

If $\mathbb{E}[X,|X| \leq y]<0$, then

$$
h_{0}:=\frac{1}{y} \log \left(1+\frac{|\mathbb{E}[X,|X| \leq y]| y^{t-1}}{\mathbb{E}\left[|X|^{t},|X| \leq y\right]}\right)
$$

is strictly positive and solves

$$
h \mathbb{E}[X,|X| \leq y]+h \frac{\mathrm{e}^{h y}-1}{y^{t-1}} \mathbb{E}\left[|X|^{t},|X| \leq y\right]=0 .
$$

Therefore, we may use Lemma 1 with $h=h_{0}$ and get an inequality with truncated moments.

To bound $\mathbb{E}\left[\mathrm{e}^{h X}, X \leq y\right]$ under the conditions of Theorem 2 we proceed similar to the proof of Theorem 3 from [15] and get

$$
\mathbb{E}\left[\mathrm{e}^{h X}, X \leq y\right] \leq 1-h a+\mathrm{e}^{t} \mathbb{E}\left[X^{2}\right] \frac{h^{2}}{2}+\frac{\mathrm{e}^{h y}-1-h y}{y^{t}} A_{t,+} .
$$


Following further the method from the proof of this theorem, we split this upper bound into two parts:

$$
\begin{gathered}
-\alpha h a+\mathrm{e}^{t} \mathbb{E}\left[X^{2}\right] \frac{h^{2}}{2}=: f_{1}(h), \\
-\beta h a+\frac{\mathrm{e}^{h y}-1-h y}{y^{t}} A_{t,+}=: f_{2}(h) .
\end{gathered}
$$

We consider $f_{1}$ and $f_{2}$ separately. It is clear that

$$
h_{1}:=\frac{2 \alpha a}{\mathrm{e}^{t} \mathbb{E}\left[X^{2}\right]}
$$

is the positive solution of the equation $f_{1}(h)=0$. Moreover, $f_{1}(h)<0$ for all $h \in\left(0, h_{1}\right)$.

Furthermore, it is easy to see that $f_{2}$ takes its unique minimum in

$$
h_{2}:=\frac{1}{y} \log \left(1+\frac{\beta a}{A_{t,+}} y^{t-1}\right) .
$$

Since $f_{2}$ is convex, we have

$$
f_{2}(h)<0 \quad \text { for all } h \in\left(0, h_{2}\right]
$$

The assumption in Theorem 2(i) means that $h_{1} \leq h_{2}$. In this case, taking into account (28), we obtain

$$
f_{1}\left(h_{1}\right)+f_{2}\left(h_{1}\right)<0 .
$$

From the latter inequality we conclude that $h_{1}$ satisfies (22) and by applying (23) with $h=h_{1}$ we obtain (9).

Under the conditions of Theorem 2(ii) we have $h_{2} \leq h_{1}$. By the same arguments we get

$$
f_{1}\left(h_{2}\right)+f_{2}\left(h_{2}\right)<0 \text {. }
$$

Then, applying (23) with $h=h_{2}$ and using the inequality $(1+u)^{x / y} \geq u^{x / y}$, we obtain (11).

\subsection{Proof of Proposition 1}

We want to use Theorem 2.1 from [4]. If we put $F:=F_{-X}$ the conditions (G1)-(G3) of this theorem are fulfilled in our setting. Hence, we get

$$
\mathbb{E}\left[R_{z}\right] \leq c \int_{0}^{\infty} \mathbb{P}(-X>u) \mathrm{d} u+c \int_{0}^{\infty} \int_{u}^{u+z} \mathbb{P}(-X>v) \mathrm{d} v \mathrm{~d} u,
$$

where

$$
c=\frac{b^{*}(\varepsilon a)}{a(1-\varepsilon)}
$$

with $b^{*}(u)=\min \{v:-\mathbb{E}[X, X<-v] \leq u\}$ and $\varepsilon \in(0,1)$ arbitrary. Clearly,

$$
\int_{0}^{\infty} \mathbb{P}(-X>u) \mathrm{d} u=\mathbb{E}[-X, X<0] .
$$


Changing the order of integration gives us

$$
\begin{aligned}
\int_{0}^{\infty} \int_{u}^{u+z} \mathbb{P}(-X>v) \mathrm{d} v \mathrm{~d} u & =\int_{0}^{z} v \mathbb{P}(-X>v) \mathrm{d} v+z \int_{z}^{\infty} \mathbb{P}(-X>v) \mathrm{d} v \\
& \leq z^{2-t} \int_{0}^{\infty} v^{t-1} \mathbb{P}(-X>v) \mathrm{d} v \\
& =\frac{z^{2-t}}{t} A_{t,-} .
\end{aligned}
$$

We easily see that,

$$
b^{*}(u) \leq\left(\frac{A_{t,-}}{u}\right)^{1 /(t-1)}
$$

therefore, by (30),

$$
c \leq \frac{A_{t,-}^{1 /(t-1)}}{a^{t /(t-1)} \varepsilon^{1 /(1-t)}(1-\varepsilon)},
$$

and, by minimisation over $\varepsilon \in(0,1)$,

$$
c \leq \frac{t^{t /(t-1)} A_{t,-}^{1 /(t-1)}}{(t-1) a^{t /(t-1)}} .
$$

Finally, combining (29), (31), (32), and (33) gives us the desired result.

\subsection{Proof of Theorem 3}

We prove (16) only. The proof of the second bound goes along the same lines.

Using Theorem 1 with $y=\theta(x+j z)$, we obtain

$$
\begin{aligned}
\mathbb{P}\left(M_{\tau_{z}}>x+j z\right) \leq & \frac{A_{t}^{1 / \theta} \theta^{-1-(t-1) / \theta} \mathbb{E}\left[\tau_{z}\right]}{a^{1 / \theta-1}(x+j z)^{1+(t-1) / \theta}} \log \left(1+\frac{a \theta^{t-1}(x+j z)^{t-1}}{A_{t}}\right) \\
& +\left(1+\frac{A_{t}^{1 / \theta} \theta^{-(t-1) / \theta}}{a^{1 / \theta}}(x+j z)^{-(t-1) / \theta}\right) \mathbb{E}\left[\tau_{z}\right] \mathbb{P}(X>\theta(x+j z)),
\end{aligned}
$$

and in view of (7),

$$
\begin{aligned}
\mathbb{P}(M>x) \leq & \frac{A_{t}^{1 / \theta} \theta^{-1-(t-1) / \theta}}{a^{1 / \theta-1}} \mathbb{E}\left[\tau_{z}\right] \Sigma_{1}(x, z) \\
& +\left(1+\frac{A_{t}^{1 / \theta} \theta^{-(t-1) / \theta}}{a^{1 / \theta}} x^{-(t-1) / \theta}\right) \mathbb{E}\left[\tau_{z}\right]\left(\mathbb{P}(X>\theta x)+\Sigma_{2}(x, z)\right),
\end{aligned}
$$

where

$$
\Sigma_{1}(x, z):=\sum_{j=0}^{\infty} \log \left(1+\frac{a \theta^{t-1}(x+j z)^{t-1}}{A_{t}}\right)(x+j z)^{-1-(t-1) / \theta}
$$

and

$$
\Sigma_{2}(x, z):=\sum_{j=1}^{\infty} \mathbb{P}(X>\theta(x+j z))
$$


Define

$$
\tilde{\Sigma}_{1}(x, z):=\sum_{j=1}^{\infty} \log \left(1+\frac{a \theta^{t-1}(x+j z)^{t-1}}{A_{t}}\right)(x+j z)^{-1-(t-1) / \theta} .
$$

The summands in this sum are strictly decreasing, so we conclude, by the integral criteria for sums that

$$
\begin{aligned}
\tilde{\Sigma}_{1}(x, z) & \leq \sum_{j=1}^{\infty} \int_{j-1}^{j} \log \left(1+\frac{a \theta^{t-1}(x+u z)^{t-1}}{A_{t}}\right)(x+u z)^{-1-(t-1) / \theta} \mathrm{d} u \\
& =\frac{1}{z} \int_{x}^{\infty} \log \left(1+\frac{a \theta^{t-1} w^{t-1}}{A_{t}}\right) w^{-1-(t-1) / \theta} \mathrm{d} w
\end{aligned}
$$

and further, by integration by parts,

$$
\begin{aligned}
& \frac{1}{z} \int_{x}^{\infty} \log \left(1+\frac{a \theta^{t-1} w^{t-1}}{A_{t}}\right) w^{-1-(t-1) / \theta} \mathrm{d} w \\
& \quad \leq \frac{\theta}{z(t-1)} \log \left(1+\frac{a \theta^{t-1} x^{t-1}}{A_{t}}\right) x^{-(t-1) / \theta}+\frac{\theta^{2}}{z(t-1)} x^{-(t-1) / \theta}
\end{aligned}
$$

Therefore, for all $x$ satisfying $x^{t-1} \geq \theta^{1-t}\left(\mathrm{e}^{\theta}-1\right) A_{t} a^{-1}$ and $x \geq z(t-1) \theta^{-1}$,

$$
\Sigma_{1}(x, z) \leq \frac{3 \theta}{z(t-1)} \log \left(1+\frac{a \theta^{t-1} x^{t-1}}{A_{t}}\right) x^{-(t-1) / \theta} .
$$

Furthermore, it is easy to see that

$$
\Sigma_{2}(x, z) \leq \sum_{j=1}^{\infty} \int_{j-1}^{j} \mathbb{P}(X>\theta(x+u z)) \mathrm{d} u=\frac{1}{\theta z} \bar{G}(\theta x),
$$

and so Theorem 3 is proved.

\subsection{Proof of Theorem 4}

Foss et al., see Theorem 5.1 of [9], have shown, for any random walk with drift $-a$ and $x_{a}$ with $x_{a} \rightarrow \infty$ as $a \rightarrow 0$, the following lower bound:

$$
\liminf _{a \rightarrow 0} \frac{\mathbb{P}\left(M^{(a)}>x_{a}\right)}{a^{-1} \bar{G}\left(x_{a}\right)} \geq 1 .
$$

It follows from the regular variation of $\mathbb{P}\left(X^{(0)}>u\right)$, that

$$
\bar{G}\left(x_{a}\right) \sim \frac{1}{r-1} x_{a}^{-r+1} L\left(x_{a}\right) \quad \text { as } a \rightarrow \infty,
$$

therefore,

$$
\mathbb{P}\left(M^{(a)} \geq x_{a}\right) \geq(1+o(1)) \frac{x_{a}^{-r+1} L\left(x_{a}\right)}{(r-1) a} \quad \text { as } a \rightarrow 0 .
$$

Thus, we only have to derive an upper bound. 
During the rest of this proof we assume $a$ to be sufficiently small in every inequality. We want to apply Theorem 3(ii) with $t<r$. It is clear that

$$
A_{t,+}^{(a)}:=\mathbb{E}\left[\left(X^{(a)}\right)^{t}, X^{(a)}>0\right] \leq \mathbb{E}\left[\left(X^{(0)}\right)^{t}, X^{(0)}>0\right]=A_{t,+}^{(0)} ;
$$

therefore, $A_{t,+}^{(a)}$ is finite for $t<r$ and

$$
\lim _{a \rightarrow 0} A_{t,+}^{(a)}=A_{t,+}^{(0)}>0 .
$$

Furthermore, we have to show that (10) is fulfilled for $y=\theta x_{a}$ under our assumptions. Since the function $y^{-1} \log \left(1+\beta a y^{t-1} / A_{t,+}^{(a)}\right)$ is decreasing for $y \gg a^{1 /(t-1)}$, we have the following bound for $x_{a} \geq c a^{-1} \log a^{-1}$ :

$$
\begin{aligned}
\frac{1}{\theta x_{a}} \log \left(1+\frac{\beta \theta^{t-1} a x_{a}^{t-1}}{A_{t,+}^{(a)}}\right) & \leq \frac{a}{\theta c \log a^{-1}} \log \left(1+\frac{\beta \theta^{t-1} c^{t-1}}{A_{t,+}^{(a)}} a^{2-t} \log ^{t-1} a^{-1}\right) \\
& =\frac{t-2}{\theta c} a(1+o(1)) .
\end{aligned}
$$

This implies that if we choose $c>\mathrm{e}^{r}(r-2) \mathbb{E}\left[X^{2}\right] / 2$ and $\theta=(1-\delta)(t-2) /(r-2)$ with $\delta$ sufficiently small, we can choose $\alpha<1$ so close to 1 that $x_{a}$ satisfies (10).

We take $z=z_{a}$ satisfying $a^{-1} \ll z \ll x_{a}$. Then, combining (12) and (13), we get

$$
\frac{\mathbb{E}\left[\tau_{z}\right]}{z} \sim \frac{1}{a} \quad \text { as } a \rightarrow 0
$$

Since $a^{-1} \ll x_{a}$ and $(t-1) / \theta-(r-1)>1 / \theta-1$ for $\theta<(t-2) /(r-2)$, we have

$$
a^{-1 / \theta+1} \frac{\mathbb{E}\left[\tau_{z}\right]}{z} \log \left(1+\frac{\beta \theta^{t-1} a x_{a}^{t-1}}{A_{t,+}^{(a)}}\right) x_{a}^{-(t-1) / \theta}=o\left(a^{-1} x_{a}^{-r+1} L\left(x_{a}\right)\right) .
$$

Furthermore, it follows from the condition $z=o\left(x_{a}\right)$ and the regular variation of $\mathbb{P}\left(X^{(0)}>x_{a}\right)$ that

$$
z \mathbb{P}\left(X^{(a)}>x_{a}\right)=o\left(x_{a}^{-r+1} L\left(x_{a}\right)\right) .
$$

Combining (36) with (38) and (35), we obtain

$$
\begin{aligned}
(1+ & \left.\left(\frac{A_{t,+}^{(a)}}{\beta \theta^{t-1} a x_{a}^{t-1}}\right)^{1 / \theta}\right) \mathbb{E}\left[\tau_{z}\right]\left(\frac{1}{\theta z} \bar{G}\left(\theta x_{a}\right)+\mathbb{P}\left(X^{(a)}>\theta x_{a}\right)\right) \\
& \sim \theta^{-r}(r-1)^{-1} a^{-1} x_{a}^{-r+1} L\left(x_{a}\right)
\end{aligned}
$$

and substituting (37) and (39) into (17) gives us

$$
\limsup _{a \rightarrow 0} \frac{\mathbb{P}\left(M^{(a)}>x_{a}\right)}{a^{-1} x_{a}^{-r+1} L\left(x_{a}\right)} \leq \theta^{-r}(r-1)^{-1} .
$$

To complete the proof it suffices to note that we can choose $\theta$ arbitrarily close to 1 by choosing $t$ close to $r$. This implies that the previous inequality is valid even with $\theta=1$. 


\subsection{Proof of Theorem 5}

We again need an upper bound only. Let $a$ be sufficiently small during this proof.

It follows from the assumptions in the theorem that $S_{n}^{(0)} / c_{n}$ converges weakly to a stable law of index $r$. The sequence $c_{n}$ can be taken from the equation $c_{n}^{-r} L\left(c_{n}\right)=1 / n$. It is known that the function $g(a)$ in the heavy-traffic approximation can be defined by the relations

$$
g(a)=1 / c_{n_{a}} \text { and } a n_{a} \sim c_{n_{a}} .
$$

The latter can be rewritten as

$$
c_{n_{a}} \sim a \frac{\left(c_{n_{a}}\right)^{r}}{L\left(c_{n_{a}}\right)} .
$$

From this we infer that (20) is equivalent to

$$
\frac{a x_{a}^{r-1}}{L\left(x_{a}\right)} \rightarrow \infty \quad \text { as } a \rightarrow 0 .
$$

We want to apply Theorem 1 for $t=2$ with $-\mathbb{E}\left[X^{(a)},\left|X^{(a)}\right| \leq y\right]$ and $A_{2}(y)$ instead of $a$ and $A_{2}$ respectively and with $y=\theta x_{a}$. According to Remark 1 we have to show that $\mathbb{E}\left[X^{(a)},\left|X^{(a)}\right| \leq \theta x_{a}\right]$ is negative. Using the Markov inequality, we have

$$
\mathbb{E}\left[X^{(a)},\left|X^{(a)}\right| \leq \theta x_{a}\right] \leq-a+\left(\theta x_{a}\right)^{-1} \mathbb{E}\left[\left(\min \left\{0, X^{(0)}\right\}\right)^{2}\right] .
$$

In view of (40), $a x_{a} \rightarrow \infty$. Therefore,

$$
\mathbb{E}\left[X^{(a)},\left|X^{(a)}\right| \leq \theta x_{a}\right] \leq-a(1+o(1)) .
$$

Furthermore,

$$
A_{2}(y) \sim \frac{r}{2-r} y^{2-r} L(y)
$$

and consequently, by $-\mathbb{E}\left[X^{(a)},\left|X^{(a)}\right| \leq \theta x_{a}\right] \sim a$, we have

$$
\begin{gathered}
A_{2}^{1 / \theta}\left(\theta x_{a}\right) \mathbb{E}\left[\tau_{z}\right] \frac{\left(-\mathbb{E}\left[X^{(a)},\left|X^{(a)}\right| \leq \theta x_{a}\right]\right)^{1-1 / \theta}}{\theta^{1+1 / \theta} x_{a}^{1+1 / \theta}} \log \left(1-\frac{\theta x_{a} \mathbb{E}\left[X^{(a)},\left|X^{(a)}\right| \leq \theta x_{a}\right]}{A_{2}\left(\theta x_{a}\right)}\right) \\
\leq(1+o(1)) k_{1} \mathbb{E}\left[\tau_{z}\right] \mathbb{P}\left(X^{(a)}>x_{a}\right) \log \left(1+k_{2} \frac{a x_{a}^{r-1}}{L\left(x_{a}\right)}\right)\left(\frac{a x_{a}^{r-1}}{L\left(x_{a}\right)}\right)^{-(1 / \theta-1)}
\end{gathered}
$$

with appropriate $k_{1}$ and $k_{2}$. Then, (40) implies that

$$
\log \left(1+c_{2} \frac{a x_{a}^{r-1}}{L\left(x_{a}\right)}\right)\left(\frac{a x_{a}^{r-1}}{L\left(x_{a}\right)}\right)^{-(1 / \theta-1)}=o(1)
$$

Furthermore,

$$
\frac{A_{2}^{1 / \theta}\left(\theta x_{a}\right)}{a^{1 / \theta}} \theta^{-1 / \theta} x_{a}^{-1 / \theta} \sim k_{3}\left(\frac{a x_{a}^{r-1}}{L\left(x_{a}\right)}\right)^{-1 / \theta}
$$

with suitable $k_{3}$ and, hence, by (40),

$$
\left(1+\frac{A_{2}^{1 / \theta}\left(\theta x_{a}\right)}{a^{1 / \theta}} \theta^{-1 / \theta} x_{a}^{-1 / \theta}\right)=(1+o(1)) .
$$


Then, combining (41), (42), and (43), Theorem 1 with $t=2$ and $y=\theta x_{a}$ gives

$$
\mathbb{P}\left(M_{\tau}^{(a)}>x_{a}\right) \leq(1+o(1)) \theta^{-r} \mathbb{E}\left[\tau_{z}\right] \mathbb{P}\left(X^{(a)}>x_{a}\right),
$$

where $\theta \in(0,1)$ is arbitrary. Hence, by $\theta \rightarrow 1$, we have

$$
\mathbb{P}\left(M_{\tau}^{(a)}>x_{a}\right) \leq(1+o(1)) \mathbb{E}\left[\tau_{z}\right] \mathbb{P}\left(X^{(a)}>x_{a}\right) .
$$

By the summation formula (7), we get a bound for the total maximum:

$$
\mathbb{P}\left(M^{(a)}>x_{a}\right) \leq(1+o(1)) \mathbb{E}\left[\tau_{z}\right] \sum_{j=0}^{\infty} \mathbb{P}\left(X^{(a)}>x_{a}+j z\right) .
$$

Combining (34) and (35) with $a^{-1} \ll z \ll x_{a}$ gives

$$
\begin{aligned}
\sum_{j=0}^{\infty} \mathbb{P}\left(X^{(a)}>x_{a}+j z\right) & \leq(1+o(1))\left(x_{a}^{-r} L\left(x_{a}\right)+\frac{x_{a}^{-r+1} L\left(x_{a}\right)}{z(r-1)}\right) \\
& \sim(1+o(1)) \frac{x_{a}^{-r+1} L\left(x_{a}\right)}{z(r-1)}
\end{aligned}
$$

and regarding (36), this completes the proof.

\section{Acknowledgement}

We are grateful to Denis Denisov for useful references.

\section{References}

[1] Asmussen, S. (1998). Subexponential asymptotics for stochastic processes: extremal behaviour, stationary distributions and first passage probabilities. Ann. Appl. Prob. 8, 354-374

[2] Asmussen, S. (2000). Ruin Probabilities. World Scientific Publishing Co., Inc., River Edge, NJ.

[3] Borovkov, A. A. (1976). Stochastic Processes in Queueing Theory. Springer, New York.

[4] Borovkov, A. A. And Foss, S. G. (2000). Estimates for overshooting an arbitrary boundary by a random walk and their applications. Theory Prob. Appl. 44, 231-253.

[5] Denisov, D. (2005). A note on the asymptotics for the maximum on a random time interval of a random walk. Markov Process. Relat. Fields 11, 165-169.

[6] Feller, W. (1971). An Introduction to Probability Theory and Its Applications, Vol. II, 2nd edn. John Wiley, New York.

[7] Foss, S. And PuhalskiI, A. A. (2011). On the limit law of a random walk conditioned to reach a high level. Stoch. Process. Appl. 121, 288-313.

[8] Foss, S. AND ZaChary, S. (2003). The maximum on a random time interval of a random walk with long-tailed increments and negative drift. Ann. Appl. Prob. 13, 37-53.

[9] Foss, S., Korshunov, D. And Zachary, S. (2011). An Introduction to Heavy-Tailed and Subexponential Distributions. Springer, New York.

[10] Fuk, D. Kh. AND Nagaev, S. V. (1971). Probability inequalities for sums of independent random variables. Theory Prob. Appl. 16, 643-660.

[11] Kalashnikov, V. (1999). Bounds for ruin probabilities in the presence of large claims and their comparison. $N$. Amer. Actuarial J. 3, 116-129.

[12] Korshunov, D. (2005). The critical case of the Cramér-Lundberg theorem on the asymptotics of the distribution of the maximum of a random walk with negative drift. Siberian Math. J. 46, 1077-1081.

[13] LoRden, G. (1970). On excess over the boundary. Ann. Math. Statist. 41, 520-527.

[14] Mogul'skil, A. A. (1974). Absolute estimates for moments of certain boundary functionals. Theory Prob. Appl. 18, 340-347.

[15] Nagaev, S. V. (1979). Large deviations of sums of independent random variables. Ann. Prob. 7, 745-789. 
[16] Olvera-Cravioto, M., Blanchet, J. and Glynn, P. (2011). On the transition from heavy traffic to heavy tails for the $M / G / 1$ queue: the regularly varying case. Ann. Appl. Prob. 21, 645-668.

[17] RichaRds, A. (2009). On upper bounds for the tail distribution of geometric sums of subexponential random variables. Queueing Systems 62, 229-242.

[18] Veraverbeke, N. (1977). Asymptotic behaviour of Wiener-Hopf factors of a random walk. Stoch. Process. Appl. 5, 27-37. 